Supporting Information for:

\title{
Cation-Anion Interactions and Polar Structures in the Solid State
}

\author{
Michael R. Marvel, ${ }^{\dagger}$ Julien Lesage,$^{\dagger}$ Jaewook Baek, ${ }^{\ddagger}$ P. Shiv Halasyamani, ${ }^{\ddagger}$ Charlotte
} L. Stern, ${ }^{\dagger}$ and Kenneth R. Poeppelmeier,

${ }^{\dagger}$ Department of Chemistry, Northwestern University, Evanston, IL 60208-3113, ‡ Department of Chemistry, University of Houston, 136 Fleming Building, Houston, TX 77204-5003

S1. Graphs of SHG responses vs. particle sizes for phase-matchable $\mathrm{LiNbO}_{3}$, non-phasematchable $\mathrm{SiO}_{2}$, and phase-matchable $\mathrm{KNaNbOF}_{5}$.

S2. X-ray diffraction data for $\mathrm{RbNaNbOF}_{5}$. The diffraction data were refined in patternmatching mode using the JADE software program in the orthorhombic space group Pbcn to lattice constants $\mathrm{a}=8.157 \AA, \mathrm{b}=13.137 \AA$, and $\mathrm{c}=10.956 \AA$. This represents a $4.8 \%$ volume decrease compared to the $\mathrm{CsNaNbOF}_{5}$ phase. A small amount of a second phase $\left(^{*}\right)$ identified as $\mathrm{Rb}_{2} \mathrm{NbOF}_{5}{ }^{1,2}$ is also present in the sample. 
Supporting Information
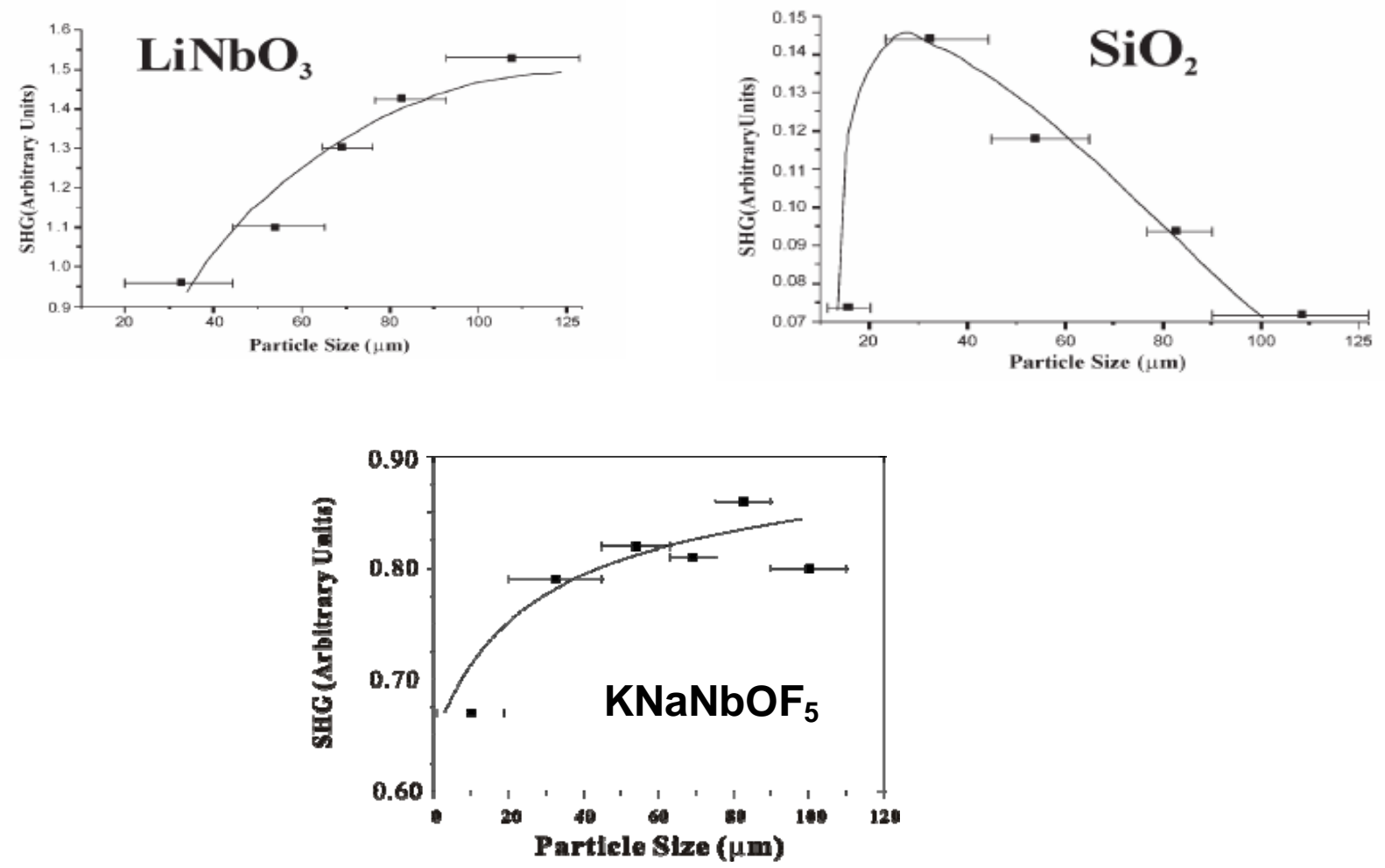

Figure S1. Graphs of SHG responses vs. particle sizes for phase-matchable $\mathrm{LiNbO}_{3}$, nonphase-matchable $\mathrm{SiO}_{2}$, and phase-matchable $\mathrm{KNaNbOF}_{5}$. 


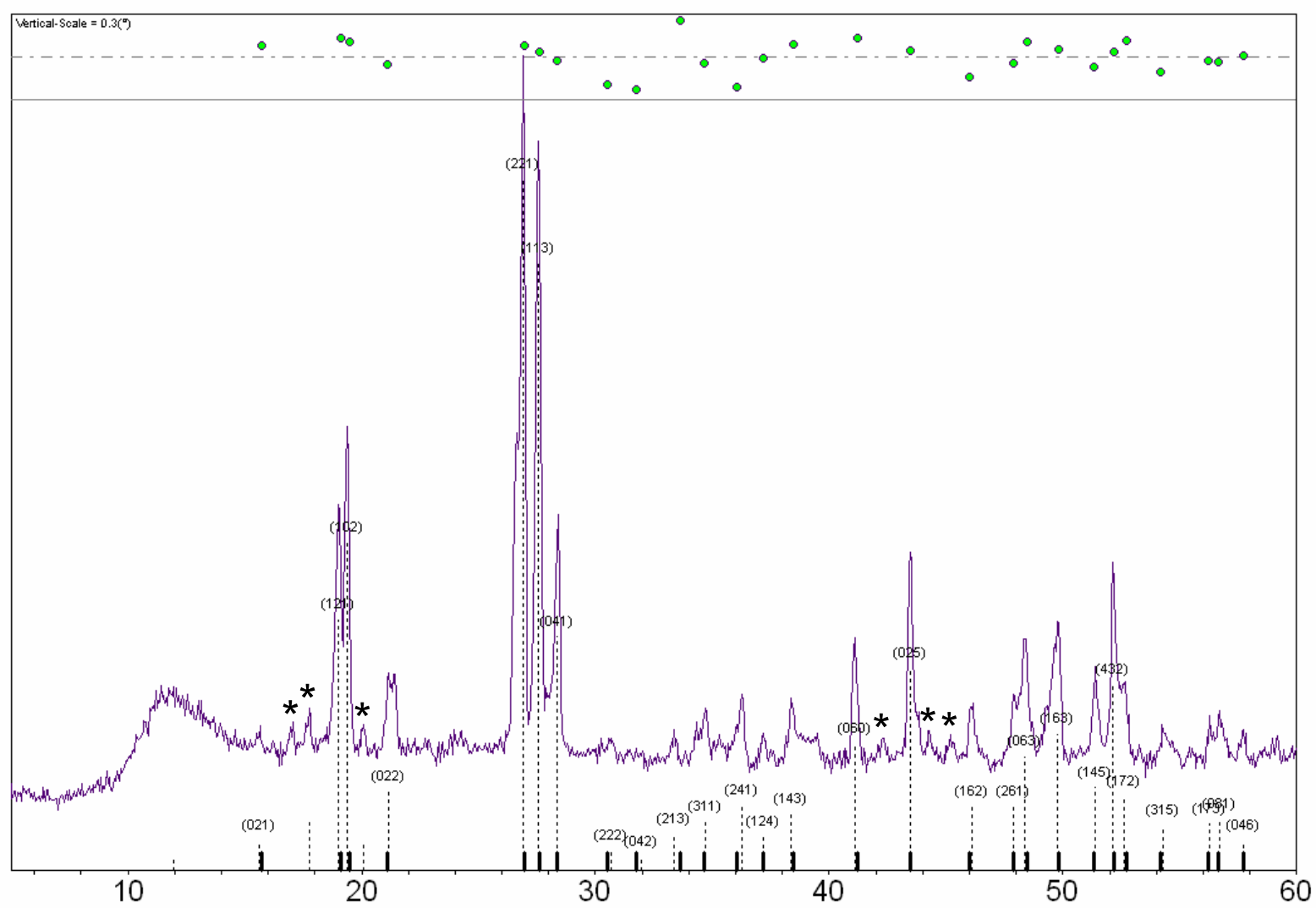

Figure S2. X-ray diffraction data for $\mathrm{RbNaNbOF}_{5}$. The diffraction data were refined in pattern-matching mode using the JADE software program in the orthorhombic space group Pbcn to lattice constants $\mathrm{a}=8.157 \AA, \mathrm{b}=13.137 \AA$, and $\mathrm{c}=10.956 \AA$. This represents a $4.8 \%$ volume decrease compared to the $\mathrm{CsNaNbOF}_{5}$ phase. A small amount of a second phase $(*)$ identified as $\mathrm{Rb}_{2} \mathrm{NbOF}_{5}{ }^{1,2}$ is also present in the sample. 


\section{References}

1. Pausewang, G., Z. Naturforsch., B: Chem. Sci. 1971, 26, 1218-20.

2. Marvel, M. R. Northwestern University, Evanston, IL. Personal Communication, 2006. 